\title{
A Pseudospectral Algorithm for Solving Multipantograph Delay Systems on a Semi-Infinite Interval Using Legendre Rational Functions
}

\author{
E. H. Doha, ${ }^{1}$ D. Baleanu, ${ }^{2,3,4}$ A. H. Bhrawy, ${ }^{5,6}$ and R. M. Hafez ${ }^{7}$ \\ ${ }^{1}$ Department of Mathematics, Faculty of Science, Cairo University, Giza 12613, Egypt \\ ${ }^{2}$ Department of Chemical and Materials Engineering, Faculty of Engineering, King Abdulaziz University, \\ Jeddah 21589, Saudi Arabia \\ ${ }^{3}$ Department of Mathematics and Computer Sciences, Cankaya University, Ankara, Turkey \\ ${ }^{4}$ Institute of Space Sciences, 76900 Magurele-Buchaarest, Romania \\ ${ }^{5}$ Department of Mathematics, Faculty of Science, King Abdulaziz University, Jeddah 21589, Saudi Arabia \\ ${ }^{6}$ Department of Mathematics, Faculty of Science, Beni-Suef University, Beni-Suef 62511, Egypt \\ ${ }^{7}$ Department of Basic Science, Institute of Information Technology, Modern Academy, Cairo 11931, Egypt
}

Correspondence should be addressed to D. Baleanu; dumitru.baleanu@gmail.com

Received 26 December 2013; Accepted 25 April 2014; Published 19 May 2014

Academic Editor: Robert A. Van Gorder

Copyright (C) 2014 E. H. Doha et al. This is an open access article distributed under the Creative Commons Attribution License, which permits unrestricted use, distribution, and reproduction in any medium, provided the original work is properly cited.

A new Legendre rational pseudospectral scheme is proposed and developed for solving numerically systems of linear and nonlinear multipantograph equations on a semi-infinite interval. A Legendre rational collocation method based on Legendre rational-Gauss quadrature points is utilized to reduce the solution of such systems to systems of linear and nonlinear algebraic equations. In addition, accurate approximations are achieved by selecting few Legendre rational-Gauss collocation points. The numerical results obtained by this method have been compared with various exact solutions in order to demonstrate the accuracy and efficiency of the proposed method. Indeed, for relatively limited nodes used, the absolute error in our numerical solutions is sufficiently small.

\section{Introduction}

Over the last three decades, the scientists have paid much attention to spectral methods due to their high accuracy (see, for instance, [1-6] and the references therein). On the other hand, spectral methods, in the context of numerical schemes for differential equations, generically belong to the family of weighted residual methods (WRMs) (cf. Finlayson [7]). WRMs represent a particular group of approximation techniques, in which the residuals (or errors) are minimized in a certain way and thereby leading to specific methods including Galerkin, Petrov-Galerkin, collocation, and tau formulations. WRMs are traditionally regarded as the foundation and cornerstone of the finite element, spectral, finite volume, boundary element, and some other methods. Many problems in science and engineering arise in unbounded domains (see, e.g., [8-12]). In general, the use of Jacobi rational functions has the advantage of obtaining the solutions in terms of the Jacobi rational parameters (see, e.g., [13-16]). Moreover, the authors of $[17,18]$ proposed an efficient collocation schemes based on the operational matrices of rational Legendre and Chebyshev functions for solving problems in the half line.

Systems of multipantograph equations model many reallife phenomena in physics and biology. Numerous numerical and analytical schemes have been presented and developed for solving such multipantograph equations, like variational iteration [19], Bernstein collocation [20], spline [21], homotopy perturbation [22], and Taylor collocation [23,24] methods. This problem has been the focus of many studies; see, for instance, [21, 25-29]. 
The main aim of this paper is to develop the spectralGauss collocation integration process based on Legendre rational functions for solving systems of linear and nonlinear multipantograph equations on the half line. The implementation of this method reduces the problem to systems of linear and nonlinear algebraic equations. The numerical simulation of multipantograph systems on a semi-infinite domain is investigated. Finally, the accuracy and applicability of the proposed method are demonstrated by test problems. Numerical results are given in which the exponential convergence behaviour is exhibited.

This paper is organized as follows. We present few revelent properties of Legendre rational functions and function approximation in the coming section. In Sections 3 and 4, the pseudospectral algorithms are implemented for solving systems of linear and nonlinear multipantograph equations. Two test examples are introduced in Section 5. Finally, some concluding remarks are given in the last section.

\section{Legendre Rational Interpolation}

In this section, we present Legendre rational functions and Legendre rational approximation that will be used to construct the Legendre rational-Gauss collocation (LR-GC) method.

2.1. Legendre Rational Functions. The well-known Legendre polynomials $L_{k}(y)$ are defined on the interval $[-1,1]$ with respect to the weight function $\rho(y)=1$. In order to use these polynomials on the interval $t \in(0, \infty)$, we recall the Legendre rational functions by introducing the change of variable $y=(t-1) /(t+1)$. Let the Legendre rational functions $L_{k}((t-1) /(t+1))$ be denoted by $R_{k}(t)$. Then $R_{k}(t)$ can be obtained with the aid of the following recurrence formula:

$$
\begin{gathered}
R_{0}=1, \quad R_{1}(t)=\frac{t-1}{t+1}, \\
R_{k+1}(t)=\left(\frac{2 k+1}{k+1}\right)\left(\frac{t-1}{t+1}\right) R_{k}(t)-\left(\frac{k}{k+1}\right) R_{k-1}(t), \\
k \geq 1 .
\end{gathered}
$$

According to the properties of the standard Legendre polynomials, we have

$$
\begin{gathered}
R_{k}(0)=(-1)^{k} \\
\partial_{t} R_{k}(t)=\frac{(k+1)}{2 t(t+1)}\left[(t-1) R_{k}(t)-(t+1) R_{k+1}(t)\right] .
\end{gathered}
$$

2.2. Function Approximation. Let $w(t)=2 /(t+1)^{2}$ denote a nonnegative, integrable, real-valued function over the interval $\Lambda=[0, \infty)$. We define

$$
L_{w}^{2}(\Lambda)=\left\{v: \Lambda \longrightarrow \mathbb{R} \mid v \text { is measurable and }\|v\|_{w}<\infty\right\},
$$

where

$$
\|v\|_{w}=\left(\int_{0}^{\infty}|v(t)|^{2} w(t) d t\right)^{1 / 2}
$$

is the norm induced by the inner product of the space $L_{w}^{2}(\Lambda)$ :

$$
\langle u, v\rangle_{w}=\int_{0}^{\infty} u(t) v(t) w(t) d t .
$$

Thus, $\left\{R_{k}(t)\right\}_{k \geq 0}$ denotes a system which is mutually orthogonal under (6); that is,

$$
\left\langle R_{k}, R_{l}\right\rangle_{w}=\frac{2}{2 k+1} \delta_{k l},
$$

where $\delta_{k l}$ is the Kronecker delta function. This system is complete in $L_{w}^{2}(\Lambda)$. For any function $u \in L_{w}^{2}(\Lambda)$ the following expansion holds:

$$
u(t)=\sum_{k=0}^{\infty} a_{k} R_{k}(t)
$$

with

$$
a_{k}=\frac{\left\langle u, R_{k}\right\rangle_{w}}{\left\|R_{k}\right\|_{w}^{2}} .
$$

2.3. Legendre Rational Interpolation Approximation. We denote by $t_{N, j}, 0 \leqslant j \leqslant N$, the nodes of the standard Legendre-Gauss interpolation on the interval $[-1,1]$, and $\omega_{N, j}, 0 \leqslant j \leqslant N$ are Christoffel numbers. The nodes of the Legendre rational-Gauss interpolation on the interval $[0, \infty)$ are the zeros of $R_{N+1}(t)$, which is denoted by $t_{R, N, j}$, $0 \leqslant j \leqslant N$. It is clear that $t_{R, N, j}=\left(1+t_{N, j}\right) /\left(1-t_{N, j}\right)$, and $\omega_{R, N, j}=\omega_{N, j}, 0 \leqslant j \leqslant N$. We now set $S_{N}(0, \infty)=$ $\operatorname{span}\left\{R_{0}(t), R_{1}(t), \ldots, R_{N}(t)\right\}$. Therefore, making use of the property of the Legendre-Gauss quadrature, we have for any $\phi \in S_{2 N+1}[0, \infty)$,

$$
\int_{0}^{\infty} w(t) \phi(t) d t=\int_{-1}^{1} \rho(y) \phi\left(\frac{1+y}{1-y}\right) d y
$$

$$
=\sum_{j=0}^{N}{\omega_{R, N, j}} \phi\left(t_{R, N, j}\right) .
$$

The interpolating function of a smooth function $u$ on a semi-infinite interval is denoted by $P_{N} u$. It is an element of $\mathfrak{R}_{N}$ and is defined as

$$
P_{N} u(t)=\sum_{0}^{N} a_{k} R_{k}(t)
$$

$P_{N} u$ is the orthogonal projection of $u$ upon $\Re_{N}$ with respect to the inner product (6) and the norm (5). Thus by the orthogonality of Legendre rational functions we have [11]

$$
\left\langle P_{N} u-u, R_{i}\right\rangle_{w}=0, \quad \forall R_{i} \in \mathfrak{R}_{N}
$$


To obtain the order of convergence of Legendre rational approximation, at first we define the space

$$
H_{w, A}^{r}(\Lambda)=\left\{v: v \text { is measurable and }\|v\|_{r, w, A}<\infty\right\},
$$

where the norm is induced by

$$
\|v\|_{r, w, A}=\left(\sum_{k=0}^{r}\left\|(t+1)^{r / 2+k} \frac{d^{k}}{d t^{k}} v\right\|_{w}^{2}\right),
$$

and $A$ is the Sturm-Liouville operator as follows:

$$
A v(t)=-w^{-1}(t) \frac{d}{d t}\left(t \frac{d}{d t} v(t)\right)
$$

We have the following theorem for the convergence.

Theorem 1. For any $v \in H_{w, A}^{r}(\Lambda)$ and $r \geq 0$,

$$
\left\|P_{N} v-v\right\| \leq c N^{-r}\|v\|_{r, w, A} .
$$

A complete proof of the theorem and discussion on convergence are given in [11].

\section{Linear Multipantograph System}

In this section, we propose the Legendre rational-Gauss collocation method to solve the following system of linear multipantograph equations:

$$
\begin{aligned}
\sum_{s=1}^{m} \beta_{1, s} u_{s}^{\prime}(t)= & \sum_{s=1}^{m} a_{1, s}(t) u_{s}(t) \\
& +\sum_{j=1}^{l} \sum_{s=1}^{m} b_{1, s}^{j}(t) u_{j}\left(\lambda_{j} t\right)+g_{1}(t), \\
\sum_{s=1}^{m} \beta_{2, s} u_{s}^{\prime}(t)= & \sum_{s=1}^{m} a_{2, s}(t) u_{s}(t) \\
& +\sum_{j=1}^{l} \sum_{s=1}^{m} b_{2, s}^{j}(t) u_{j}\left(\lambda_{j} t\right)+g_{2}(t) \\
& 0<t \leq \infty, \\
& +\sum_{j=1}^{l} \sum_{s=1}^{m} b_{m, s}^{j}(t) u_{j}\left(\lambda_{j} t\right)+g_{m}(t), \\
\sum_{s=1}^{m} \beta_{m, s} u_{s}^{\prime}(t)= & \sum_{s=1}^{m} a_{m, s}(t) u_{s}(t)
\end{aligned}
$$

subject to

$$
\begin{gathered}
\sum_{r=1}^{m} u_{r, 1}(0)=u_{1}, \\
\sum_{r=1}^{m} u_{r, 2}(0)=u_{2}, \\
\vdots \\
\sum_{r=1}^{m} u_{r, m}(0)=u_{m} .
\end{gathered}
$$

Let us first introduce some basic notation that will be used in the sequel. We define the discrete inner product and norm as follows:

$$
\begin{gathered}
(u, v)_{w, N}=\sum_{j=0}^{N} u\left(t_{R, N, j}\right) v\left(t_{R, N, j}\right) \omega_{R, N, j}, \\
\|u\|_{w, N}=\sqrt{(u, u)_{w, N}} .
\end{gathered}
$$

Obviously,

$$
(u, v)_{w, N}=(u, v)_{w}, \quad \forall u, v \in S_{2 N+1} .
$$

The Legendre rational-Gauss collocation method for solving (17) and (18) is to seek $u_{j, N}(t) \in S_{N}(0, \infty), j=1,2, \ldots, k$, such that

$$
\begin{aligned}
& \sum_{s=1}^{m} \beta_{1, s} u_{s}^{\prime}\left(t_{q, R, N, \gamma}\right)=\sum_{s=1}^{m} a_{1, s}(t) u_{s}\left(t_{q, R, N, \gamma}\right) \\
& +\sum_{j=1}^{l} \sum_{s=1}^{m} b_{1, s}^{j}(t) u_{j}\left(\lambda_{j} t_{q, R, N, \gamma}\right) \\
& +g_{1}\left(t_{q, R, N, \gamma}\right) \text {, } \\
& \sum_{s=1}^{m} \beta_{2, s} u_{s}^{\prime}\left(t_{q, R, N, \gamma}\right)=\sum_{s=1}^{m} a_{2, s}(t) u_{s}\left(t_{q, R, N, \gamma}\right) \\
& +\sum_{j=1}^{l} \sum_{s=1}^{m} b_{2, s}^{j}(t) u_{j}\left(\lambda_{j} t_{q, R, N, \gamma}\right) \\
& +g_{2}\left(t_{q, R, N, \gamma}\right) \text {, } \\
& \sum_{s=1}^{m} \beta_{m, s} u_{s}^{\prime}\left(t_{q, R, N, \gamma}\right)=\sum_{s=1}^{m} a_{m, s}(t) u_{s}\left(t_{q, R, N, \gamma}\right) \\
& +\sum_{j=1}^{l} \sum_{s=1}^{m} b_{m, s}^{j}(t) u_{j}\left(\lambda_{j} t_{q, R, N, \gamma}\right) \\
& +g_{m}\left(t_{q, R, N, \gamma}\right) \text {, } \\
& q=1,2, \ldots, m, \quad \gamma=0,1, \ldots, N-1,
\end{aligned}
$$




$$
\begin{gathered}
\sum_{r=1}^{m} u_{r, 1}(0)=u_{1} \\
\sum_{r=1}^{m} u_{r, 2}(0)=u_{2} \\
\vdots \\
\sum_{r=1}^{m} u_{r, m}(0)=u_{m}
\end{gathered}
$$$$
\sum_{h=0}^{N} \sum_{s=1}^{m} \beta_{2, s} \mathcal{c}_{2, h} \frac{(h+1)}{2 t(t+1)}\left[(t-1) R_{s, h}(t)-(t+1) R_{s, h+1}(t)\right]
$$$$
=\sum_{h=0}^{N} \sum_{s=1}^{m} a_{2, s}(t) c_{2, h} R_{s, h}(t)+\sum_{h=0}^{N} \sum_{j=1}^{l} \sum_{s=1}^{m} b_{2, s}^{j}(t) c_{2, j} R_{s, j}\left(\lambda_{j} t\right)
$$$$
+g_{2}(t)
$$

We derive the algorithm for solving (17)-(18). To do this, let

$$
u_{i, N}(t)=\sum_{h=0}^{N} c_{i, h} R_{i, h}(t)
$$

Now, substitution of (22) into (17) enables us to write

$$
\begin{aligned}
\sum_{h=0}^{N} \sum_{s=1}^{m} \beta_{1, s} c_{1, h} D R_{s, h}(t) & =\sum_{h=0}^{N} \sum_{s=1}^{m} a_{1, s}(t) c_{1, h} R_{s, h}(t) \\
& +\sum_{h=0}^{N} \sum_{j=1}^{l} \sum_{s=1}^{m} b_{1, s}^{j}(t) c_{1, j} R_{s, j}\left(\lambda_{j} t\right) \\
& +g_{1}(t), \\
& \sum_{h=0}^{N} \sum_{s=1}^{m} a_{2, s}(t) c_{2, h} R_{s, h}(t) \\
\sum_{h=0}^{N} \sum_{s=1}^{m} \beta_{2, s} \mathcal{c}_{2, h} D R_{s, h}(t) & \sum_{h=0}^{N} \sum_{j=1}^{l} \sum_{s=1}^{m} b_{2, s}^{j}(t) c_{2, j} R_{s, j}\left(\lambda_{j} t\right) \\
& +g_{2}(t), \\
& \vdots \\
\sum_{h=0}^{N} \sum_{s=1}^{m} \beta_{m, s} c_{m, h} D R_{s, h}(t)= & \sum_{h=0}^{N} \sum_{s=1}^{m} a_{m, s}(t) c_{m, h} R_{s, h}(t) \\
& +\sum_{h=0}^{N} \sum_{j=1}^{l} \sum_{s=1}^{m} b_{m, s}^{j}(t) c_{m, j} R_{s, j}\left(\lambda_{j} t\right) \\
& +g_{m}(t)
\end{aligned}
$$

$$
\begin{gathered}
\sum_{h=0}^{N} \sum_{r=1}^{m}(-1)^{h} a_{1, h}=u_{1}, \\
\sum_{h=0}^{N} \sum_{r=1}^{m}(-1)^{h} a_{2, h}=u_{2}, \\
\vdots \\
\sum_{h=0}^{N} \sum_{r=1}^{m}(-1)^{h} a_{m, h}=u_{m} .
\end{gathered}
$$

Then, by virtue of (3), we deduce that

$$
\begin{aligned}
\sum_{h=0}^{N} & \sum_{s=1}^{m} \beta_{1, s} c_{1, h} \frac{(h+1)}{2 t(t+1)}\left[(t-1) R_{s, h}(t)-(t+1) R_{s, h+1}(t)\right] \\
= & \sum_{h=0}^{N} \sum_{s=1}^{m} a_{1, s}(t) c_{1, h} R_{s, h}(t)+\sum_{h=0}^{N} \sum_{j=1}^{l} \sum_{s=1}^{m} b_{1, s}^{j}(t) c_{1, j} R_{s, j}\left(\lambda_{j} t\right) \\
& +g_{1}(t),
\end{aligned}
$$

Moreover, the initial condition (18)-with the aid of (2)yields

$$
\begin{aligned}
= & \sum_{h=0}^{N} \sum_{s=1}^{m} a_{m, s}(t) c_{m, h} R_{s, h}(t)+\sum_{h=0}^{N} \sum_{j=1}^{l} \sum_{s=1}^{m} b_{m, s}^{j}(t) c_{m, j} R_{s, j}\left(\lambda_{j} t\right) \\
& +g_{m}(t) .
\end{aligned}
$$




$$
\begin{aligned}
\sum_{h=0}^{N} \sum_{s=1}^{m} \beta_{2, s} c_{2, h} \frac{(h+1)}{2 t_{q, R, N, \gamma}\left(t_{q, R, N, \gamma}+1\right)} & \times \\
\times & {\left[\left(t_{q, R, N, \gamma}-1\right) R_{s, h}\left(t_{q, R, N, \gamma}\right)\right.} \\
& \left.\quad-\left(t_{q, R, N, \gamma}+1\right) R_{s, h+1}\left(t_{q, R, N, \gamma}\right)\right] \\
= & \sum_{h=0}^{N} \sum_{s=1}^{m} a_{2, s}\left(t_{q, R, N, \gamma}\right) c_{2, h} R_{s, h}\left(t_{q, R, N, \gamma}\right) \\
+ & \sum_{h=0}^{N} \sum_{j=1}^{l} \sum_{s=1}^{m} b_{2, s}^{j}\left(t_{q, R, N, \gamma}\right) c_{2, j} R_{s, j}\left(\lambda_{j} t_{q, R, N, \gamma}\right) \\
+ & g_{2}\left(t_{q, R, N, \gamma}\right)
\end{aligned}
$$$$
\sum_{h=0}^{N} \sum_{s=1}^{m} \beta_{m, s} c_{m, h} \frac{(h+1)}{2 t_{q, R, N, \gamma}\left(t_{q, R, N, \gamma}+1\right)}
$$$$
\times\left[\left(t_{q, R, N, \gamma}-1\right) R_{s, h}\left(t_{q, R, N, \gamma}\right)\right.
$$$$
\left.-\left(t_{q, R, N, \gamma}+1\right) R_{s, h+1}\left(t_{q, R, N, \gamma}\right)\right]
$$$$
=\sum_{h=0}^{N} \sum_{s=1}^{m} a_{m, s}\left(t_{q, R, N, \gamma}\right) c_{m, h} R_{s, h}\left(t_{q, R, N, \gamma}\right)
$$$$
+\sum_{h=0}^{N} \sum_{j=1}^{l} \sum_{s=1}^{m} b_{m, s}^{j}\left(t_{q, R, N, \gamma}\right) c_{m, j} R_{s, j}\left(\lambda_{j} t_{q, R, N, \gamma}\right)
$$$$
+g_{m}\left(t_{q, R, N, \gamma}\right) \text {. }
$$

Thus (26) with relation (25) generate $(N+1)$ of a set of algebraic equations which can be solved for the unknown coefficients $c_{i, j},(i=1, \ldots, m ; j=0,1,2, \ldots, N)$, by using any standard solver technique.

\section{Nonlinear Multipantograph System}

In this section, we consider the nonlinear multipantograph system of the form

$$
\begin{array}{r}
\sum_{s=1}^{m} \xi_{1, s} u_{s}^{\prime}(t)=F\left(t, \sum_{s=1}^{m} \zeta_{1, s} u_{s}(t), \sum_{s=1}^{m} \eta_{1, s} u_{s}(\lambda t)\right), \\
\sum_{s=1}^{m} \xi_{2, s} u_{s}^{\prime}(t)=F\left(t, \sum_{s=1}^{m} \zeta_{2, s} u_{s}(t), \sum_{s=1}^{m} \eta_{2, s} u_{s}(\lambda t)\right), \\
t \in(0, \infty) \text { where } \lambda \in(0,1), \\
\vdots \\
\sum_{s=1}^{m} \xi_{m, s} u_{s}^{\prime}(t)=F\left(t, \sum_{s=1}^{m} \zeta_{m, s} u_{s}(t), \sum_{s=1}^{m} \eta_{m, s} u_{s}(\lambda t)\right),
\end{array}
$$

with initial conditions

$$
\begin{gathered}
\sum_{r=1}^{m} u_{r, 1}(0)=u_{1}, \\
\sum_{r=1}^{m} u_{r, 2}(0)=u_{2}, \\
\vdots \\
\sum_{r=1}^{m} u_{r, m}(0)=u_{m} .
\end{gathered}
$$

The Legendre rational-Gauss collocation method for solving (27) and (28) is to seek $u_{i, N}(x) \in S_{N}(0, \infty)$, such that

$$
\begin{array}{r}
\sum_{s=1}^{m} \xi_{1, s} u_{s, N}^{\prime}(t)=F\left(t, \sum_{s=1}^{m} \zeta_{1, s} u_{s, N}(t), \sum_{s=1}^{m} \eta_{1, s} u_{s, N}(\lambda t)\right), \\
\sum_{s=1}^{m} \xi_{2, s} u_{s, N}^{\prime}(t)=F\left(t, \sum_{s=1}^{m} \zeta_{2, s} u_{s, N}(t), \sum_{s=1}^{m} \eta_{2, s} u_{s, N}(\lambda t)\right), \\
t \in(0, \infty) \text { where } \lambda \in(0,1), \\
\vdots \\
\sum_{s=1}^{m} \xi_{m, s} u_{s, N}^{\prime}(t)=F\left(t, \sum_{s=1}^{m} \zeta_{m, s} u_{s, N}(t), \sum_{s=1}^{m} \eta_{m, s} u_{s, N}(\lambda t)\right)
\end{array}
$$

is satisfied exactly at the collocation points $t_{R, N, k}, k=$ $0,1, \ldots, N-1$. In other words, we have to collocate (29) at the $(N)$ Legendre rational roots $t_{R, N, k}$, which immediately yields

$$
\begin{gathered}
\sum_{h=0}^{N} \sum_{s=1}^{m} \xi_{m, s} c_{1, j} D R_{s, h}\left(t_{q, R, N, k}\right) \\
=F\left(t_{q, R, N, k}, \sum_{h=0}^{N} \sum_{s=1}^{m} \zeta_{1, s} \mathcal{c}_{1, h} R_{s, h}\left(t_{q, R, N, k}\right),\right. \\
\left.\sum_{h=0}^{N} \sum_{s=1}^{m} \eta_{1, s} \mathcal{c}_{1, h} R_{s, h}\left(\lambda t_{q, R, N, k}\right)\right), \\
\sum_{h=0}^{N} \sum_{s=1}^{m} \xi_{m, s} \mathcal{C}_{2, j} D R_{s, h}\left(t_{q, R, N, k}\right) \\
=F\left(t_{q, R, N, k}, \sum_{h=0}^{N} \sum_{s=1}^{m} \zeta_{2, s} \mathcal{c}_{2, h} R_{s, h}\left(t_{q, R, N, k}\right),\right. \\
\left.\sum_{h=0}^{N} \sum_{s=1}^{m} \eta_{2, s} \mathcal{C}_{2, h} R_{s, h}\left(\lambda t_{q, R, N, k}\right)\right),
\end{gathered}
$$




$$
\begin{gathered}
\sum_{h=0}^{N} \sum_{s=1}^{m} \xi_{m, s} c_{m, j} D R_{s, h}\left(t_{q, R, N, k}\right) \\
=F\left(t_{q, R, N, k}, \sum_{h=0}^{N} \sum_{s=1}^{m} \zeta_{m, s} c_{m, h} R_{s, h}\left(t_{q, R, N, k}\right),\right. \\
\left.\sum_{h=0}^{N} \sum_{s=1}^{m} \eta_{m, s} c_{m, h} R_{s, h}\left(\lambda t_{q, R, N, k}^{(\theta, 9)}\right)\right),
\end{gathered}
$$

with (28) written in the form

$$
\begin{gathered}
\sum_{h=0}^{N} \sum_{r=1}^{m} c_{1, j} R_{r, h}(0)=u_{1,0}, \\
\sum_{h=0}^{N} \sum_{r=1}^{m} c_{2, j} R_{r, h}(0)=u_{2,0}, \\
\vdots \\
\sum_{h=0}^{N} \sum_{r=1}^{m} c_{m, j} R_{r, h}(0)=u_{n, 0} .
\end{gathered}
$$

This constitute a system of $(N+1)$ nonlinear algebraic equations in the unknown expansion coefficients $c_{i, j},(i=$ $1, \ldots, m ; j=0,1,2, \ldots, N)$, which can be solved by using any standard iteration technique, like Newton's iteration method.

\section{Numerical Results}

In this section, we present two numerical examples in order to show the accuracy of Legendre rational collocation method for solving multipantograph delay system. The following tables and figures contain the values of the exact solutions $u_{i}(x), i=1,2, \ldots, k$, the approximate solutions $u_{i, N}(x), i=$ $1,2, \ldots, k$, and the absolute error functions $e_{i, N}(x)=\mid u_{i}(x)-$ $u_{i, N}(x) \mid, i=1,2, \ldots, k$, at the selected points of the given interval.

Example 1. Consider the following linear multipantograph delay system:

$$
\begin{array}{r}
u_{1}^{\prime}(t)+5 u_{2}^{\prime}(t)+t u_{1}(t)-2 t^{2} u_{2}(t) \\
+u_{1}(0.5 t)+t u_{2}(0.8 t)=g_{1}(t), \\
0 \leq t \leq 100, \\
-4 u_{1}^{\prime}(t)+u_{2}^{\prime}(t)-u_{1}(t)+t u_{2}(t) \\
+t u_{1}(0.2 t)+u_{2}(0.4 t)=g_{2}(t),
\end{array}
$$

with the initial conditions

$$
u_{1}(0)=0, \quad u_{2}^{\prime}(0)=1,
$$

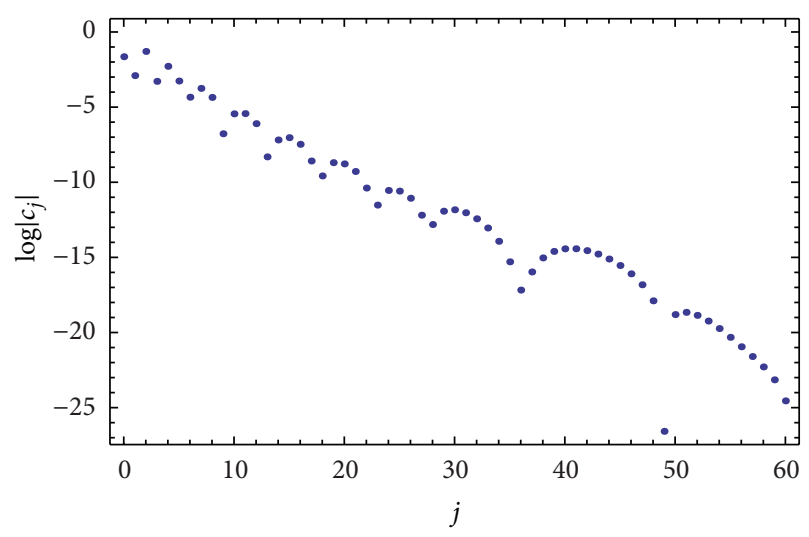

FIGURE 1: Logarithmic graph of absolute coefficients $\left|c_{j}\right|$ of Legendre rational functions at $N=60$ of Example 1 .

TABLE 1: Comparison of the absolute errors $e_{1, N}$ for $u_{1}(t)$ of (32).

\begin{tabular}{lccc}
\hline$t_{i}$ & & LR-GC method & \\
& $N=20$ & $N=40$ & $N=60$ \\
\hline 0.0 & $0.000 \times 10^{+00}$ & $0.000 \times 10^{+00}$ & $2.775 \times 10^{-17}$ \\
10.0 & $1.105 \times 10^{-4}$ & $5.638 \times 10^{-8}$ & $3.779 \times 10^{-10}$ \\
20.0 & $2.175 \times 10^{-5}$ & $4.347 \times 10^{-7}$ & $2.609 \times 10^{-9}$ \\
30.0 & $3.087 \times 10^{-3}$ & $6.613 \times 10^{-7}$ & $8.111 \times 10^{-9}$ \\
40.0 & $1.080 \times 10^{-2}$ & $6.894 \times 10^{-7}$ & $1.604 \times 10^{-8}$ \\
50.0 & $2.036 \times 10^{-2}$ & $1.644 \times 10^{-6}$ & $4.074 \times 10^{-8}$ \\
60.0 & $3.008 \times 10^{-2}$ & $6.281 \times 10^{-6}$ & $9.717 \times 10^{-8}$ \\
70.0 & $3.922 \times 10^{-2}$ & $1.594 \times 10^{-5}$ & $1.190 \times 10^{-7}$ \\
80.0 & $4.756 \times 10^{-2}$ & $3.053 \times 10^{-5}$ & $8.903 \times 10^{-8}$ \\
90.0 & $5.506 \times 10^{-2}$ & $4.921 \times 10^{-5}$ & $4.396 \times 10^{-8}$ \\
100.0 & $6.178 \times 10^{-2}$ & $7.100 \times 10^{-5}$ & $3.624 \times 10^{-8}$ \\
\hline
\end{tabular}

where

$$
\begin{aligned}
& g_{1}(t)=2 t-4 t^{2}+\left(3 t^{2}-t+6\right) e^{-t}+0.5 t e^{-0.5 t}-t e^{-0.8 t} \\
& g_{2}(t)=2+2 t+(2 t-3) e^{-t}-e^{-0.4 t}+0.2 t^{2} e^{-0.2 t}
\end{aligned}
$$

The exact solution of the system is $u_{1}(t)=t e^{-t}$ and $u_{2}(t)=$ $2-e^{-t}$.

Tables 1 and 2 list the results obtained by the Legendre rational-Gauss collocation method in terms of maximum absolute errors with different values of $N$. The logarithmic graphs of absolute coefficients for Legendre rational functions are shown in Figures 1 and 2. This confirms that the proposed method has reasonable convergence rate. 
TABLE 2: Comparison of the absolute errors $e_{2 N}$ for $u_{2}(t)$ of (32).

\begin{tabular}{lccc}
\hline$t_{i}$ & & LR-GC method & \\
& $N=20$ & $N=40$ & $N=60$ \\
\hline 0.0 & $8.273 \times 10^{-7}$ & $9.056 \times 10^{-12}$ & $4.393 \times 10^{-11}$ \\
10.0 & $1.395 \times 10^{-5}$ & $6.763 \times 10^{-9}$ & $4.703 \times 10^{-12}$ \\
20.0 & $9.412 \times 10^{-6}$ & $8.911 \times 10^{-9}$ & $5.661 \times 10^{-11}$ \\
30.0 & $6.376 \times 10^{-5}$ & $1.177 \times 10^{-8}$ & $1.242 \times 10^{-10}$ \\
40.0 & $1.499 \times 10^{-4}$ & $7.887 \times 10^{-9}$ & $2.575 \times 10^{-10}$ \\
50.0 & $2.121 \times 10^{-4}$ & $1.621 \times 10^{-8}$ & $3.371 \times 10^{-10}$ \\
60.0 & $2.529 \times 10^{-4}$ & $4.544 \times 10^{-8}$ & $6.993 \times 10^{-10}$ \\
70.0 & $2.791 \times 10^{-4}$ & $1.027 \times 10^{-7}$ & $9.146 \times 10^{-10}$ \\
80.0 & $2.957 \times 10^{-4}$ & $1.820 \times 10^{-7}$ & $7.991 \times 10^{-10}$ \\
90.0 & $3.061 \times 10^{-4}$ & $2.729 \times 10^{-7}$ & $5.234 \times 10^{-10}$ \\
100.0 & $3.125 \times 10^{-4}$ & $3.658 \times 10^{-7}$ & $3.478 \times 10^{-10}$ \\
\hline
\end{tabular}

Example 2. Consider the following nonlinear multipantograph delay system:

$$
\begin{aligned}
u_{1}^{\prime}(t)+2 u_{2}^{\prime}(t)+u_{1}(t)+t u_{2}(t)+t u_{2}^{2}(0.5 t) & =g_{1}(t), \\
0 & \leq t \leq 1, \\
3 u_{1}^{\prime}(t)-u_{2}^{\prime}(t)+t u_{1}^{2}(0.2 t)-(t+1) u_{2}(t) & =g_{2}(t),
\end{aligned}
$$

with the initial conditions

$$
u_{1}(0)=0, \quad u_{2}^{\prime}(0)=1,
$$

where

$$
\begin{aligned}
g_{1}(t)= & -2 e^{-t} \sin (t)-e^{-t} \cos (t) \\
& +e^{-t} t \cos (t)+e^{-t} t \cos ^{2}(0.5 t), \\
g_{2}(t)= & 3 e^{-t} \cos (t)-2 e^{-t} \sin (t) \\
& -t e^{-t} \sin (t)+e^{-0.4 t} \sin ^{2}(0.2 t) .
\end{aligned}
$$

The exact solution of the system is $u_{1}(x)=e^{-t} \sin (t)$ and $u_{2}(t)=e^{-t} \cos (t)$.

In Tables 3 and 4, we list the absolute errors obtained by the Legedre rational-Gauss collocation method, with different values of $N$. Figures 3 and 4 are plotted to compare the analytic solution with the approximate solution at $N=28$.

\section{Conclusion}

In this paper, a collocation Legendre rational method has been proposed to obtain the approximate solutions of systems of multipantograph delay equations. The derivation of this method is essentially based on Legendre rational functions and Gauss quadrature formula. The main advantage of the developed method is that high accurate solutions were achieved using few numbers of the Legendre rational functions. Additionally, if $N$ is increased, it can be seen that approximate solutions obtained by the method are close to the exact solutions.

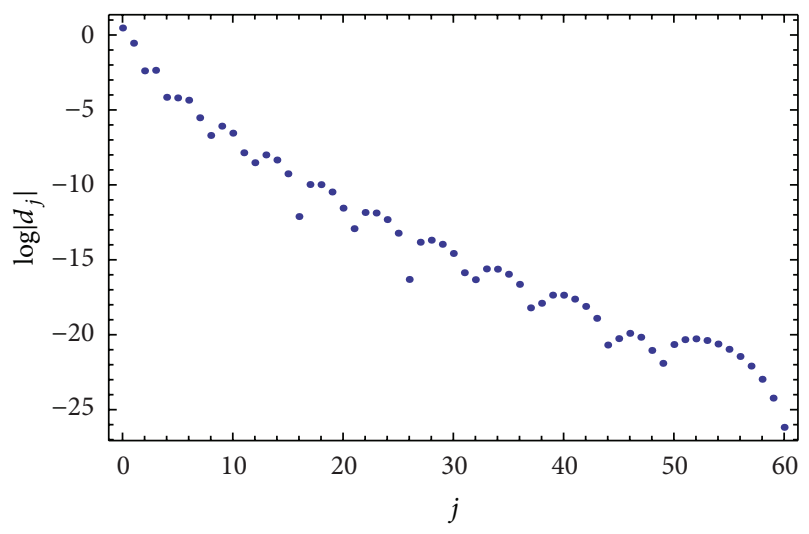

FigURE 2: Logarithmic graph of absolute coefficients $\left|d_{j}\right|$ of Legendre rational functions at $N=60$ of Example 1 .

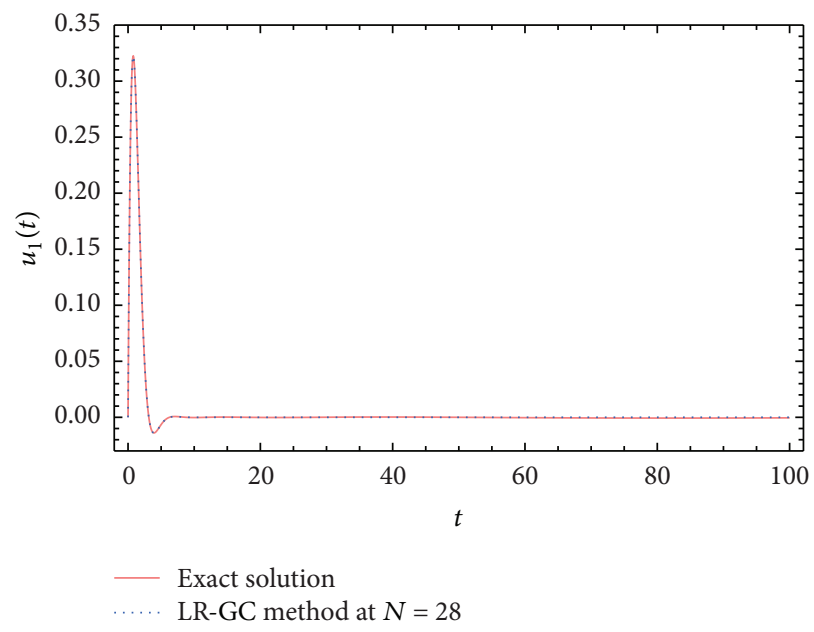

FIGURE 3: Graph of exact solution and approximate solution $u_{1}(t)$ at $N=28$ for Example 2 .

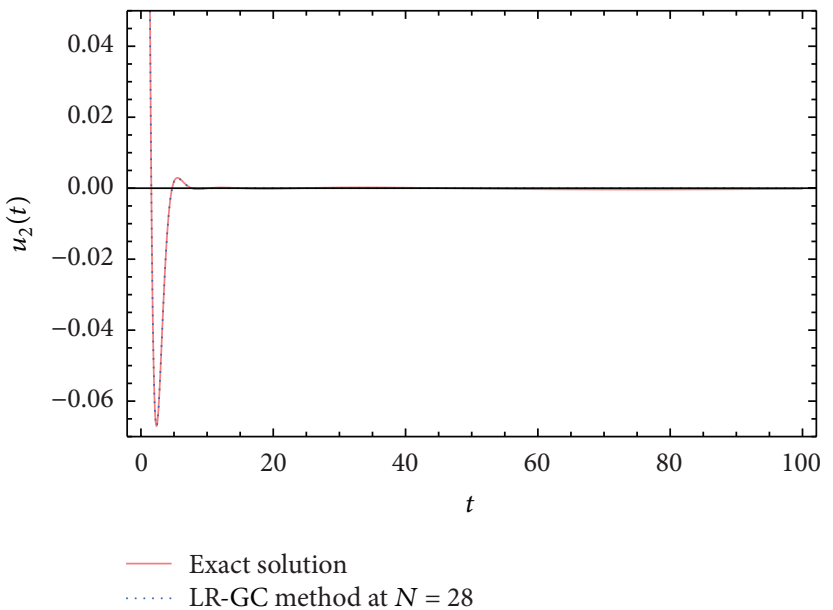

FIGURE 4: Graph of exact solution and approximate solution $u_{2}(t)$ at $N=28$ for Example 2 . 
TABLE 3: Comparison of the absolute errors $e_{1, N}$ for $u_{1}(t)$ of (35).

\begin{tabular}{lccc}
\hline$t_{i}$ & & LR-GC method & \\
& $N=10$ & $N=30$ & $N=50$ \\
\hline 0.0 & $5.551 \times 10^{-17}$ & $0.000 \times 10^{+00}$ & $0.000 \times 10^{+00}$ \\
1.0 & $1.554 \times 10^{-3}$ & $4.799 \times 10^{-6}$ & $8.262 \times 10^{-8}$ \\
2.0 & $2.085 \times 10^{-3}$ & $2.349 \times 10^{-6}$ & $4.957 \times 10^{-8}$ \\
3.0 & $6.583 \times 10^{-3}$ & $1.392 \times 10^{-6}$ & $1.069 \times 10^{-7}$ \\
4.0 & $1.408 \times 10^{-3}$ & $9.788 \times 10^{-9}$ & $1.247 \times 10^{-7}$ \\
5.0 & $5.932 \times 10^{-3}$ & $2.183 \times 10^{-5}$ & $8.508 \times 10^{-7}$ \\
6.0 & $6.690 \times 10^{-3}$ & $6.643 \times 10^{-5}$ & $1.169 \times 10^{-6}$ \\
7.0 & $3.789 \times 10^{-3}$ & $6.919 \times 10^{-6}$ & $1.485 \times 10^{-6}$ \\
8.0 & $1.092 \times 10^{-3}$ & $6.729 \times 10^{-5}$ & $6.698 \times 10^{-7}$ \\
9.0 & $1.546 \times 10^{-3}$ & $2.765 \times 10^{-5}$ & $1.382 \times 10^{-6}$ \\
10.0 & $2.137 \times 10^{-4}$ & $2.345 \times 10^{-5}$ & $1.450 \times 10^{-6}$ \\
\hline
\end{tabular}

TABLE 4: Comparison of the absolute errors $e_{2, N}$ for $u_{2}(t)$ of (35).

\begin{tabular}{lccc}
\hline$t_{i}$ & & LR-GC method & \\
& $N=10$ & $N=30$ & $N=50$ \\
\hline 0.0 & $0.000 \times 10^{+00}$ & $1.110 \times 10^{-16}$ & $1.665 \times 10^{-16}$ \\
1.0 & $6.260 \times 10^{-4}$ & $2.819 \times 10^{-6}$ & $5.391 \times 10^{-8}$ \\
2.0 & $6.997 \times 10^{-5}$ & $2.048 \times 10^{-5}$ & $4.171 \times 10^{-8}$ \\
3.0 & $5.800 \times 10^{-5}$ & $1.042 \times 10^{-6}$ & $9.088 \times 10^{-8}$ \\
4.0 & $6.901 \times 10^{-4}$ & $7.814 \times 10^{-6}$ & $1.541 \times 10^{-7}$ \\
5.0 & $2.427 \times 10^{-3}$ & $9.524 \times 10^{-6}$ & $2.014 \times 10^{-7}$ \\
6.0 & $3.012 \times 10^{-3}$ & $1.126 \times 10^{-6}$ & $1.116 \times 10^{-7}$ \\
7.0 & $1.483 \times 10^{-3}$ & $1.283 \times 10^{-5}$ & $2.553 \times 10^{-7}$ \\
8.0 & $8.598 \times 10^{-3}$ & $4.171 \times 10^{-6}$ & $5.499 \times 10^{-7}$ \\
9.0 & $1.529 \times 10^{-2}$ & $6.713 \times 10^{-6}$ & $2.949 \times 10^{-7}$ \\
10.0 & $1.995 \times 10^{-2}$ & $3.503 \times 10^{-5}$ & $3.311 \times 10^{-7}$ \\
\hline
\end{tabular}

\section{Conflict of Interests}

The authors declare that there is no conflict of interests regarding the publication of this paper.

\section{Acknowledgments}

This paper was funded by the Deanship of Scientific Research DSR, King Abdulaziz University, Jeddah. The authors, therefore, acknowledge with thanks DSR technical and financial support.

\section{References}

[1] C. Canuto, M. Y. Hussaini, A. Quarteroni, and T. A. Zang, Spectral Methods: Fundamentals in Single Domains, Springer, New York, NY, USA, 2006.

[2] A. H. Bhrawy and M. A. Alghamdi, "A shifted Jacobi-GaussLobatto collocation method for solving nonlinear fractional Langevin equation involving two fractional orders in different intervals," Boundary Value Problems, vol. 2012, article 62, 2012.
[3] A. H. Bhrawy, "A Jacobi-Gauss-Lobatto collocation method for solving generalized Fitzhugh-Nagumo equation with timedependent coefficients," Applied Mathematics and Computation, vol. 222, pp. 255-264, 2013.

[4] E. H. Doha, A. H. Bhrawy, M. A. Abdelkawy, and R. A. Van Gorder, "Jacobi-Gauss-Lobatto collocation method for the numerical solution of $1+1$ nonlinear Schrödinger equations," Journal of Computational Physics, vol. 261, pp. 244-255, 2014.

[5] A. B. Koc, M. Cakmak, A. Kurnaz, and K. Uslu, "A new Fibonacci type collocation procedure for boundary value problems," Advances in Difference Equations, vol. 2013, article 262, 2013.

[6] Y. Simsek, "Unification of the Bernstein-type polynomials and their applications," Boundary Value Problems, vol. 2013, article 56, 2013.

[7] B. A. Finlayson, The Method of Weighted Residuals and Variational Principles, Academic Press, New York, NY, USA, 1972.

[8] D. Funaro, "Computational aspects of pseudospectral Laguerre approximations," Applied Numerical Mathematics, vol. 6, no. 6, pp. 447-457, 1990.

[9] B.-y. Guo, "Jacobi approximations in certain Hilbert spaces and their applications to singular differential equations," Journal of Mathematical Analysis and Applications, vol. 243, no. 2, pp. 373408, 2000.

[10] J. P. Boyd, Chebyshev and Fourier Spectral Methods, Dover, New York, NY, USA, 2nd edition, 2000.

[11] B.-Y. Guo, J. Shen, and Z.-Q. Wang, "A rational approximation and its applications to differential equations on the half line," Journal of Scientific Computing, vol. 15, no. 2, pp. 117-147, 2000.

[12] B.-Y. Guo, J. Shen, and Z.-Q. Wang, "Chebyshev rational spectral and pseudospectral methods on a semi-infinite interval," International Journal for Numerical Methods in Engineering, vol. 53, no. 1, pp. 65-84, 2002.

[13] E. H. Doha, A. H. Bhrawy, R. M. Hafez, and R. A. Van Gorder, "A Jacobi rational pseudospectral method for Lane-Emden initial value problems an astrophysics on a semi-infinite interval," Computational and Applied Mathematics, 2013.

[14] E. H. Doha, A. H. Bhrawy, D. Baleanu, and R. M. Hafez, "A new Jacobi rational-Gauss collocation method for numerical solution of generalized pantograph equations," Applied Numerical Mathematics, vol. 77, pp. 43-54, 2014.

[15] E. H. Doha, A. H. Bhrawy, R. M. Hafez, and R. A. Van Gorder, "Jacobi rational-Gauss collocation method for Lane-Emden equations of astrophysical significance," Nonlinear Analysis: Modelling and Control. In press.

[16] Y.-G. Yi and B.-Y. Guo, "Generalized Jacobi rational spectral method on the half line," Advances in Computational Mathematics, vol. 37, no. 1, pp. 1-37, 2012.

[17] K. Parand, M. Shahini, and M. Dehghan, "Rational Legendre pseudospectral approach for solving nonlinear differential equations of Lane-Emden type," Journal of Computational Physics, vol. 228, no. 23, pp. 8830-8840, 2009.

[18] K. Parand and M. Razzaghi, "Rational Chebyshev tau method for solving higher-order ordinary differential equations," International Journal of Computer Mathematics, vol. 81, no. 1, pp. 7380, 2004.

[19] X. Chen and L. Wang, "The variational iteration method for solving a neutral functional-differential equation with proportional delays," Computers \& Mathematics with Applications, vol. 59, no. 8, pp. 2696-2702, 2010. 
[20] O. R. Işik, Z. Güney, and M. Sezer, "Bernstein series solutions of pantograph equations using polynomial interpolation," Journal of Difference Equations and Applications, vol. 18, no. 3, pp. 357374, 2012.

[21] A. El-Safty and S. M. Abo-Hasha, "On the application of spline functions to initial value problems with retarded argument," International Journal of Computer Mathematics, vol. 32, pp. 173$179,1990$.

[22] J. Biazar and B. Ghanbari, “The homotopy perturbation method for solving neutral functional-differential equations with proportional delays," Journal of King Saud University: Science, vol. 24, no. 1, pp. 33-37, 2012.

[23] M. Sezer, S. Yalçinbaş, and N. Şahin, "Approximate solution of multi-pantograph equation with variable coefficients," Journal of Computational and Applied Mathematics, vol. 214, no. 2, pp. 406-416, 2008

[24] M. Gülsu and M. Sezer, "A Taylor collocation method for solving high-order linear pantograph equations with linear functional argument," Numerical Methods for Partial Differential Equations, vol. 27, no. 6, pp. 1628-1638, 2011.

[25] Y. Kuang, Delay Differential Equations with Applications in Population Dynamics, vol. 191, Academic Press, New York, NY, USA, 1993.

[26] D. J. Evans and K. R. Raslan, "The Adomian decomposition method for solving delay differential equation," International Journal of Computer Mathematics, vol. 82, no. 1, pp. 49-54, 2005.

[27] E. Tohidi, A. H. Bhrawy, and K. Erfani, "A collocation method based on Bernoulli operational matrix for numerical solution of generalized pantograph equation," Applied Mathematical Modelling, vol. 37, no. 6, pp. 4283-4294, 2013.

[28] Z.-H. Yu, "Variational iteration method for solving the multipantograph delay equation," Physics Letters. A, vol. 372, no. 43, pp. 6475-6479, 2008.

[29] S. Widatalla, "A comparative study on the stability of Laplaceadomian algorithm and numerical methods in generalized pantograph equations," ISRN Computational Mathematics, vol. 2012, Article ID 704184, 6 pages, 2012. 


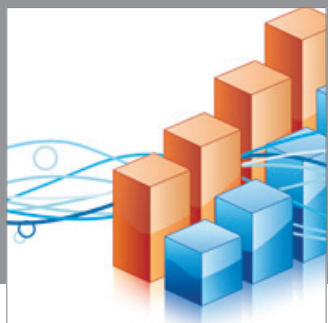

Advances in

Operations Research

mansans

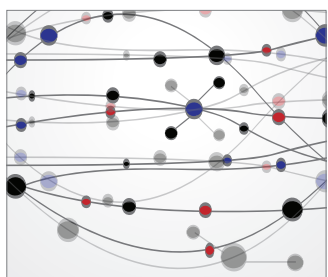

The Scientific World Journal
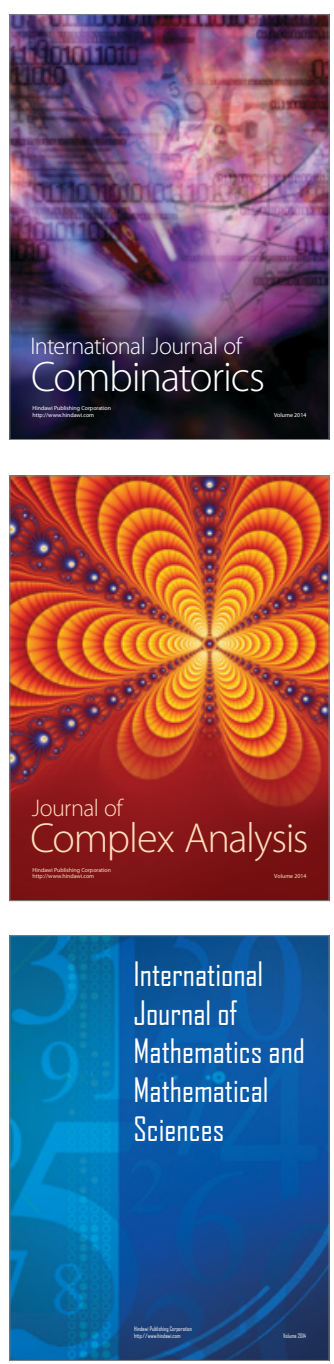
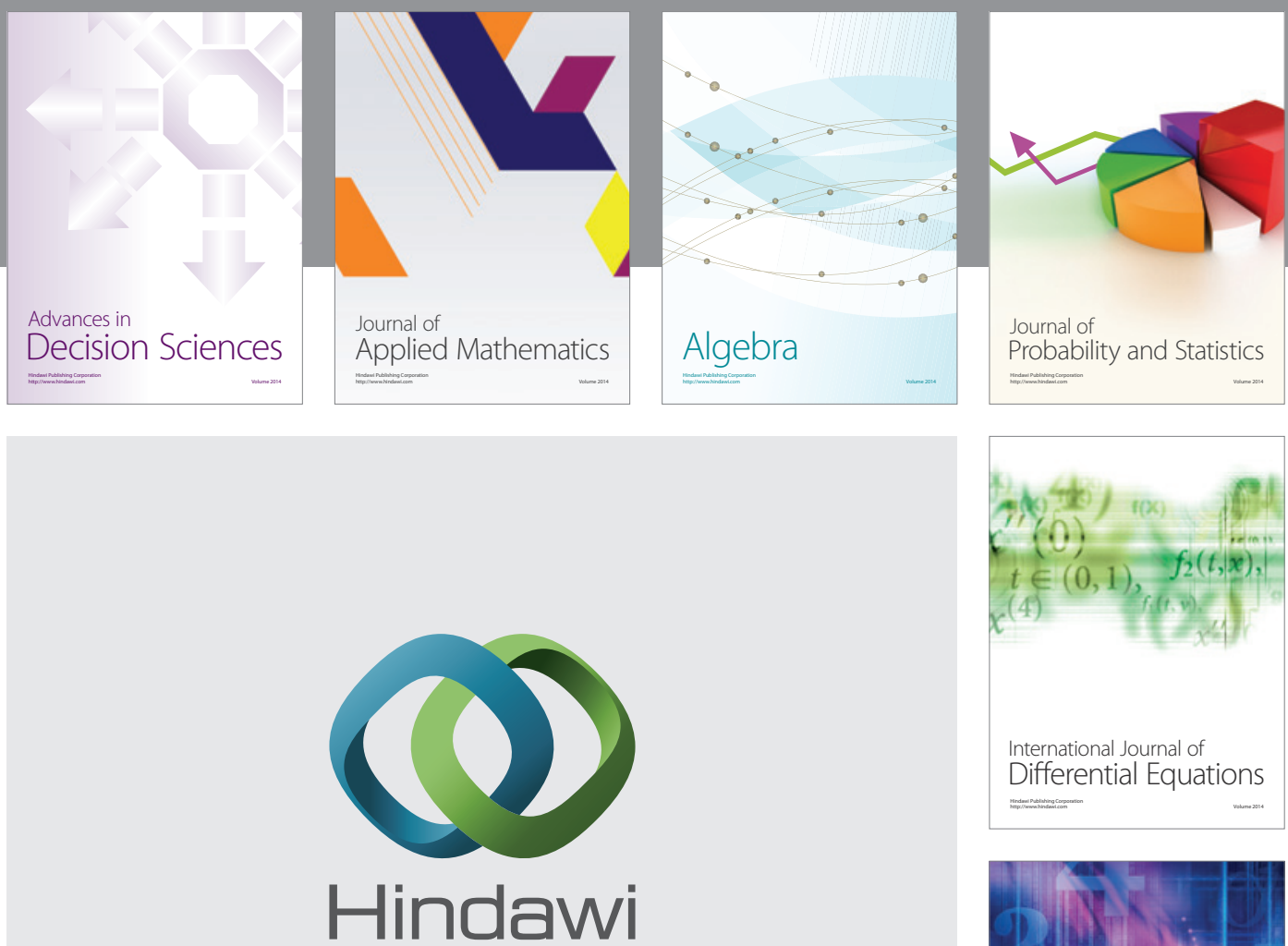

Submit your manuscripts at http://www.hindawi.com
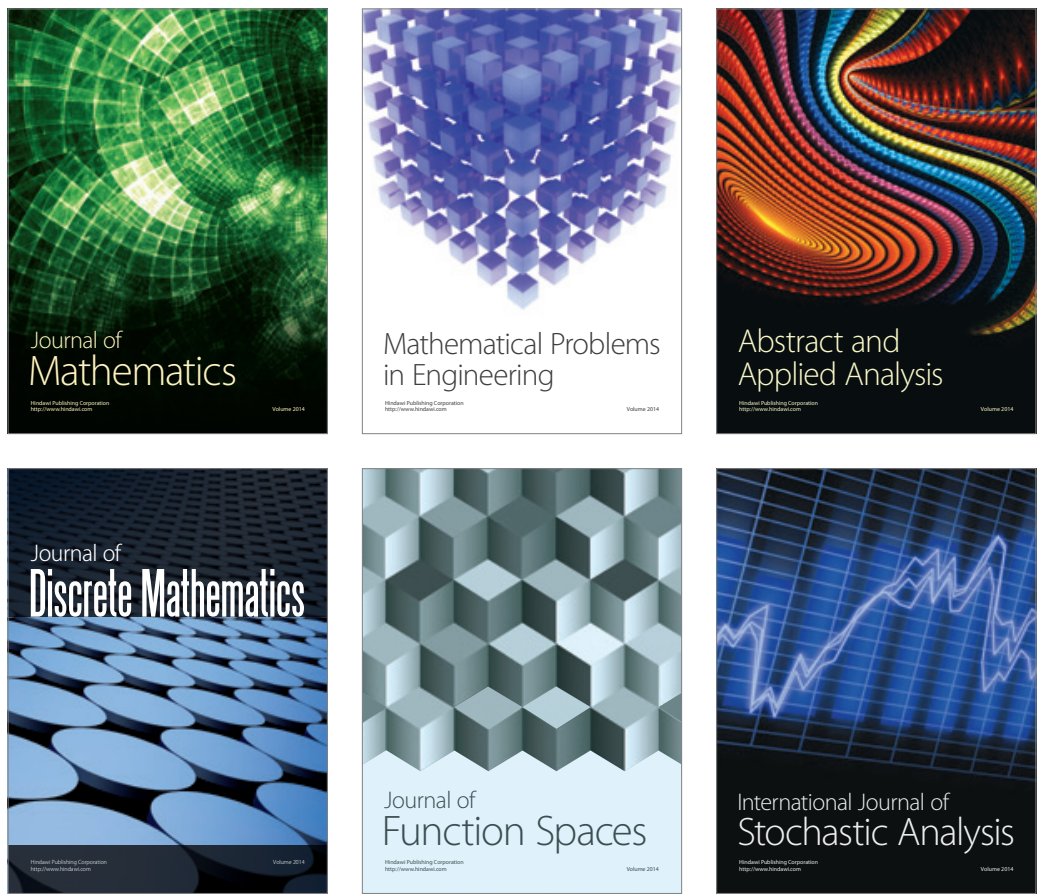

Journal of

Function Spaces

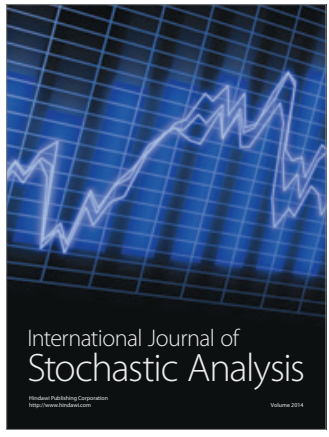

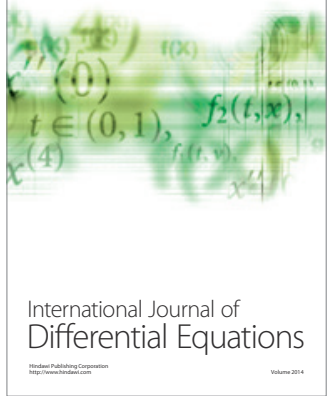
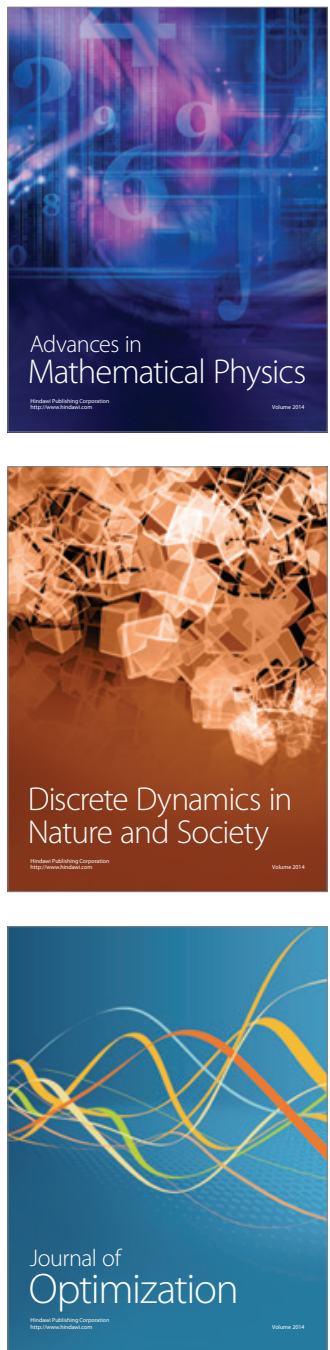